\title{
Evidence for broad versus segregated projections from cholinergic and noradrenergic nuclei to functionally and anatomically discrete subregions of prefrontal cortex
}

\author{
Daniel Chandler and Barry D. Waterhouse* \\ Department of Neurobiology and Anatomy, Drexel University College of Medicine, Philadelphia, PA, USA
}

\section{Edited by:}

Anita Disney, Salk Institute for

Biological Studies, USA

\section{Reviewed by:}

René Hurlemann, University of Bonn, Germany

Elisabeth Van Bockstaele, Thomas

Jefferson University, USA

\section{${ }^{*}$ Correspondence:}

Barry D. Waterhouse, Department of Neurobiology and Anatomy, Drexel

University College of Medicine, 2900

Queen Lane, Philadelphia, PA 19129,

USA.

e-mail: barry.waterhouse@

drexelmed.edu

\begin{abstract}
The prefrontal cortex (PFC) is implicated in a variety of cognitive and executive operations. However, this region is not a single functional unit; rather, it is composed of several functionally and anatomically distinct networks, including anterior cingulate cortex (ACC), medial prefrontal cortex (mPFC), and orbitofrontal cortex (OFC). These prefrontal subregions serve dissociable behavioral functions, and are unique in their afferent and efferent connections. Each of these subregions is innervated by ascending cholinergic and noradrenergic systems, each of which likewise has a distinct role in cognitive function; yet the distribution and projection patterns of cells in the source nuclei for these pathways have not been examined in great detail. In this study, fluorescent retrograde tracers were injected into ACC, mPFC, and OFC, and labeled cells were identified in the cholinergic nucleus basalis of Meynert (NBM) and noradrenergic nucleus locus coeruleus (LC). Injections into all three cortical regions consistently labeled cells primarily ipsilateral to the injection site with a minimal contralateral component. In NBM, retrogradely labeled neurons were scattered throughout the rostral half of the nucleus, whereas those in LC tended to cluster in the core of the nucleus, and were rarely localized within the rostral or caudal poles. In NBM, more than half of all retrogradely labeled cells possessed axon collaterals projecting two or more PFC subregions. In LC, however, only $4.3 \%$ of retrogradely labeled neurons possessed collaterals targeting any two prefrontal subregions simultaneously, and no cells were identified that projected to all three regions. Of all labeled LC neurons, $49.3 \%$ projected only to mPFC, $28.5 \%$ projected only to OFC, and $18.0 \%$ projected only to ACC. These findings suggest that subsets of LC neurons may be capable of modulating neuronal activity in individual prefrontal subregions independently, whereas assemblies of NBM cells may exert a more unified influence on the three areas, simultaneously. This work emphasizes unique aspects of the cholinergic and noradrenergic projections to functionally and anatomically distinct subregions of PFC and provides insights regarding global versus segregated regulation of prefrontal operations by these neuromodulatory pathways.
\end{abstract}

Keywords: orbitofrontal cortex, medial prefrontal cortex, anterior cingulate cortex, nucleus basalis of Meynert, locus coeruleus, prefrontal cortex, norepinephrine, acetylcholine
The prefrontal cortex (PFC) is critically involved in the maintenance of cognitive and executive functions (Dias et al., 1996, 1997; Birrell and Brown, 2000; Robbins, 2000; Dalley et al., 2004). In both rodent and primate (including humans), distinct subregions of PFC have been shown to mediate distinct behaviors and cognitive roles (Owen et al., 1991, 1993; Dias et al., 1996, 1997; Birrell and Brown, 2000; McGaughy et al., 2008; Newman and McGaughy, 2008). Accumulating evidence implicates anterior cingulate cortex (ACC), medial prefrontal cortex (mPFC), and orbitofrontal cortex (OFC) in distinct behavioral and cognitive functions such that restricted region specific lesions each produce distinct behavioral and cognitive abnormalities. Therefore, dysfunction of these, and other, prefrontal subregions and their associated circuitries, likely contribute to the manifestation of cognitive symptoms associated with neuropsychiatric and neurodegenerative disease (Pantelis et al., 1999; Arnsten, 2000; Dalley et al., 2004; Rahman et al., 2006; Ramos and Arnsten, 2007).

In rodents, lesions of ACC produce characteristic impulsive behaviors such as premature responding and over-responsiveness to non-relevant stimuli in several behavioral tasks (Muir et al., 1996; Bussey et al., 1997a,b; Parkinson et al., 2000; Cardinal et al., 2002). In non-human primates, lesions of the dorsolateral PFC, but not OFC, produce impairments in extradimensional shifting, a behavior characterized by switching attention from one perceptual dimension to another (Dalley et al., 2004). Similar observations have been made for mPFC and OFC in rodent (Birrell and Brown, 2000; Bissonette et al., 2008; McGaughy et al., 2008; Newman et al., 2008). Conversely, in both rodents and nonhuman primates, lesions of OFC, but not mPFC or dorsolateral PFC impairs performance in reversal learning, a class of behavioral 
tasks that requires attentional switching without changing attention to perceptual dimension (Dias et al., 1996, 1997; Dalley et al., 2004; Bissonette et al., 2008). Therefore, ACC lesions produce impulsive behavior, responding to task-irrelevant, and distractor stimuli, whereas mPFC and OFC lesions produce perseverative behavior and an inability to dissociate attention from stimuli that have lost their behavioral relevance. OFC, however, seems to facilitate lower-order discriminations within the same perceptual dimension, whereas mPFC promotes higher order discriminations, allowing an animal to shift attention to novel unrelated stimulus features (Dalley et al., 2004). The roles of these three subregions in the healthy brain therefore collectively contribute to the maintenance of cognitive and executive function, permitting an organism to properly allocate attentional reserves in a complex and dynamic world. These functions become perturbed in a number of neuropsychiatric and neurodegenerative diseases; therefore it is critically important to understand the underlying modulatory mechanisms of these behaviors.

Several neuromodulatory pathways converge in PFC, including cholinergic input from the nucleus basalis of Meynert (NBM) and noradrenergic input from the pontine nucleus locus coeruleus (LC). The NBM is the primary source of acetylcholine (ACh) in the cerebral cortex (Wenk, 1997; Sarter and Bruno, 2000), has a prominent role in arousal, learning, attention, and memory (Wenk, 1997; McGaughy and Sarter, 1998, 1999; McGaughy et al., 2000; Sarter and Bruno, 2000; Nieto-Escamez et al., 2002), and undergoes neuronal degeneration in Alzheimer's disease (Lehmann et al., 1980; Arendt et al., 1983; Pang et al., 1993). By contrast, the LC is the only source of norepinephrine (NE) to the forebrain and PFC (Berridge and Waterhouse, 2003; Sara, 2009) and modulates sensory processing, motor behavior, arousal, and cognitive processes (McGaughy and Sarter, 1998; Devilbiss and Waterhouse, 2000, 2004; Berridge and Waterhouse, 2003; Hurley et al., 2004; Devilbiss et al., 2006; Moxon et al., 2007; McGaughy et al., 2008; Newman et al., 2008; Sara, 2009; Cain et al., 2011). A loss of LC neurons occurs in Alzheimer's and Parkinson's diseases (Bondareff et al., 1982; Gesi et al., 2000; Von Coelln et al., 2004; McMillan et al., 2011), which may be related to the cognitive decline associated with the progression of these disease processes. Given the importance of PFC integrity in maintaining cognition and executive function, it seems likely that the PFC projection neurons in NBM and LC may be those that become dysfunctional or degenerate in various psychiatric and neurodegenerative diseases.

Both noradrenergic and cholinergic systems are extensively targeted by pharmacological therapeutics designed to treat symptoms of psychiatric and neurodegenerative disease (Robbins, 2000; Milstein et al., 2007; Arnsten and Pliszka, 2011; Gamo and Arnsten, 2011; Wallace and Porter, 2011; Wallace et al., 2011). However, evidence suggests that these systems serve dissociable functions within the cognitive realm (McGaughy et al., 2008). Data from computational modeling (Yu and Dayan, 2002, 2005) and behavioral paradigms (Chiba et al., 1995; McGaughy and Sarter, 1995; Bucci et al., 1998; Robbins, 2000; Dalley et al., 2001, 2004; Bouret and Sara, 2004, 2005; Maddux et al., 2007) suggest that cholinergic transmission signals "expected uncertainty," whereas noradrenergic transmission signals "unexpected uncertainty," For example, in a go/no-go task in which an animal must fixate its vision on a central point and indicate if specific target stimulus (such as a red square) appears in a random location on a screen, there is expected uncertainty in the location that the target will appear: the animal has been trained to know that the target will appear, but where this will occur is uncertain. Such conditions have been shown to recruit the cholinergic system (Yu and Dayan, 2002, 2005). However, if a distractor stimulus (such as a blue circle) is also present and likewise appears randomly in different locations on the screen, unexpected uncertainty occurs if the red square suddenly loses its relevance and the blue circle instead becomes the target. These type of unexpected changes recruit the noradrenergic system, which is needed to promote behavioral adaptation to the rule change that the animal had not previously learned (Robbins, 2000; Dalley et al., 2001, 2004; McGaughy et al., 2008; Cain et al., 2011). Importantly, these neurochemical specific effects are also region specific: noradrenergic denervation of mPFC impairs extradimensional shifting (McGaughy et al., 2008; Newman et al., 2008), a behavior which requires adaptation to unexpected uncertainty, but not reversal learning. Furthermore, although a NE-specific lesion produces this effect, cholinergic denervation of mPFC does not (McGaughy et al., 2008). A loss of cholinergic input to mPFC, on the other hand, impairs behavior in the five choice serial reaction time task, in which an animal must divide its attention between five randomly flashing stimulus lights, and correctly report which light flashed to retrieve reward (Dalley et al., 2001, 2004; McGaughy et al., 2002; Maddux et al., 2007). The cholinergic and noradrenergic systems in PFC, therefore, are requisite for behavioral adaptation to both expected and unexpected certainty, and permit an animal to appropriately allocate attentional reserves and navigate in a complex and dynamic world.

Little attention has been paid, however, to the organization of the efferent projections of the NBM and LC to OFC, mPFC and ACC. A rough efferent topography of the cortical projection from NBM has been identified in primate: rostral and medial groups of cells project to the medial surface of the cortex, rostral and lateral groups project to frontal and parietal cortices and amygdala, intermediate regions project to prefrontal, insular, and posterior parietal cortices, and caudal portions project to the superior and temporal cortex (Pang et al., 1993). This nucleus is less well defined and its efferent cholinergic neurons are more scattered in the rodent (Wenk, 1997; Sarter and Bruno, 2000); however, it similarly stains intensely for cholinergic markers, exists within the substantia innominata of the basal forebrain, and has likewise been implicated in the modulation of higher order cognitive processes (Lehmann et al., 1980; Wenk, 1997; McGaughy and Sarter, 1998, 1999; Sarter and Bruno, 2000; Nieto-Escamez et al., 2002). Previous reports of LC anatomy suggest that this nucleus is highly divergent with only modest efferent topography (Fallon and Loughlin, 1982; Loughlin et al., 1982; Waterhouse et al., 1983, 1993), although some neurons have a tendency to collateralize to target multiple structures along the same sensory pathway (Simpson et al., 1997, 1999, 2006). The nature of the projections from LC and NBM to the subregions of PFC, however, has not yet been explored. Because of the prominent role of PFC in normal cognitive processes and the absence or compromise of these facilities in neurodegenerative and neuropsychiatric disease, it is critical to have specific knowledge of these afferent modulatory pathways. 
Studies from our laboratory are currently underway to determine the organization of the monoaminergic and cholinergic projections from NBM and LC to discrete subregions of PFC. The results reported here summarize the findings of experiments targeting the NBM and LC and show that OFC, mPFC, and ACC are differentially innervated by discrete groupings of cells in these nuclei. In these studies, adult male Sprague-Dawley rats (Taconic, $n=10$ ) underwent a surgical procedure to pressure inject each region with $0.3 \mu \mathrm{l}$ of different fluorescently labeled retrograde tracers (FluoroEmerald, FluoroRuby, Cascade Blue dextran, 10,000 MW, Invitrogen) at a flow rate of $0.036 \mu \mathrm{l} / \mathrm{min}$ using a motorized syringe pump. Therefore, all animals in the study received a differently labeled tracer into each region, such that all were injected a total of three times. In this way, the prefrontal terminal field(s) of individual retrogradely labeled cells in NBM and LC could be determined. Injections were counterbalanced between surgeries to control for regional differences in uptake and transport of the different tracers. After a one week survival time, rats were perfused and brains were extracted and sectioned at $80 \mu \mathrm{m}$ (injection sites) or $30 \mu \mathrm{m}$ (NBM and LC). Sections containing NBM and LC underwent fluorescent immunostains for the vesicular acetylcholine transporter (VAChT) and dopamine $\beta$ hydroxylase (DBH), respectively, to identify the borders of and cell types in each nucleus. Tissue containing injection sites underwent a fluorescent Nissl stain and were microscopically observed to determine if tracer diffused beyond the anatomical boundaries of each PFC subregion. The injected volumes were identical for all tracers and all regions within and between animals. All tracers in all regions diffused similar distances from the site of deposition and filled all cortical layers. Any cases in which tracer diffused beyond the anatomical boundaries of OFC, mPFC, and ACC, or between these regions $(n=1)$ were excluded from further analysis. Sections were visualized on a Leica DMBRE fluorescence microscope with filters to detect FluoroRuby labeled cells (ex/em = 540/605), FluoroEmerald labeled cells $(\mathrm{ex} / \mathrm{em}=480 / 535)$ Cascade Blue labeled cells $(\mathrm{ex} / \mathrm{em}=405 / 460)$ and VAChT/DBH immunoreactive cells $(\mathrm{ex} / \mathrm{em}=620 / 700)$ so that the terminal field(s) and putative neurochemical identity of each retrogradely labeled cell could be identified. Fluorescent photomicrographs were generated using a Retiga EXi camera and QImaging software. Figure 1 shows representative injections into OFC, $\mathrm{MPFC}$, and ACC of a single animal. Black and white low power images in Figures 2A and 3A show representative sections of NBM and LC through the fluorescent filter used to detect VAChT and $\mathrm{DBH}$ immunoreactivity, respectively. Color photomicrographs in Figures 2B and 3B show merged images of the same section photographed through individual fluorescence filters that were used to detect each tracer. All neurons in LC express DBH and therefore all retrogradely labeled cells in LC were included in the analysis. However, not all retrogradely labeled cells in the region of NBM stained positively for VAChT; as such, only those cells that were immunoreactive for the peptide were counted. High power photomicrographs in Figures 2C and 3C show retrogradely labeled cells denoted by arrows and Roman numerals in low power images as viewed through each fluorescence filter to detect retrograde tracers (rows 1-3), the merged image (row 4), and the fluorescent immunostain (row 5).

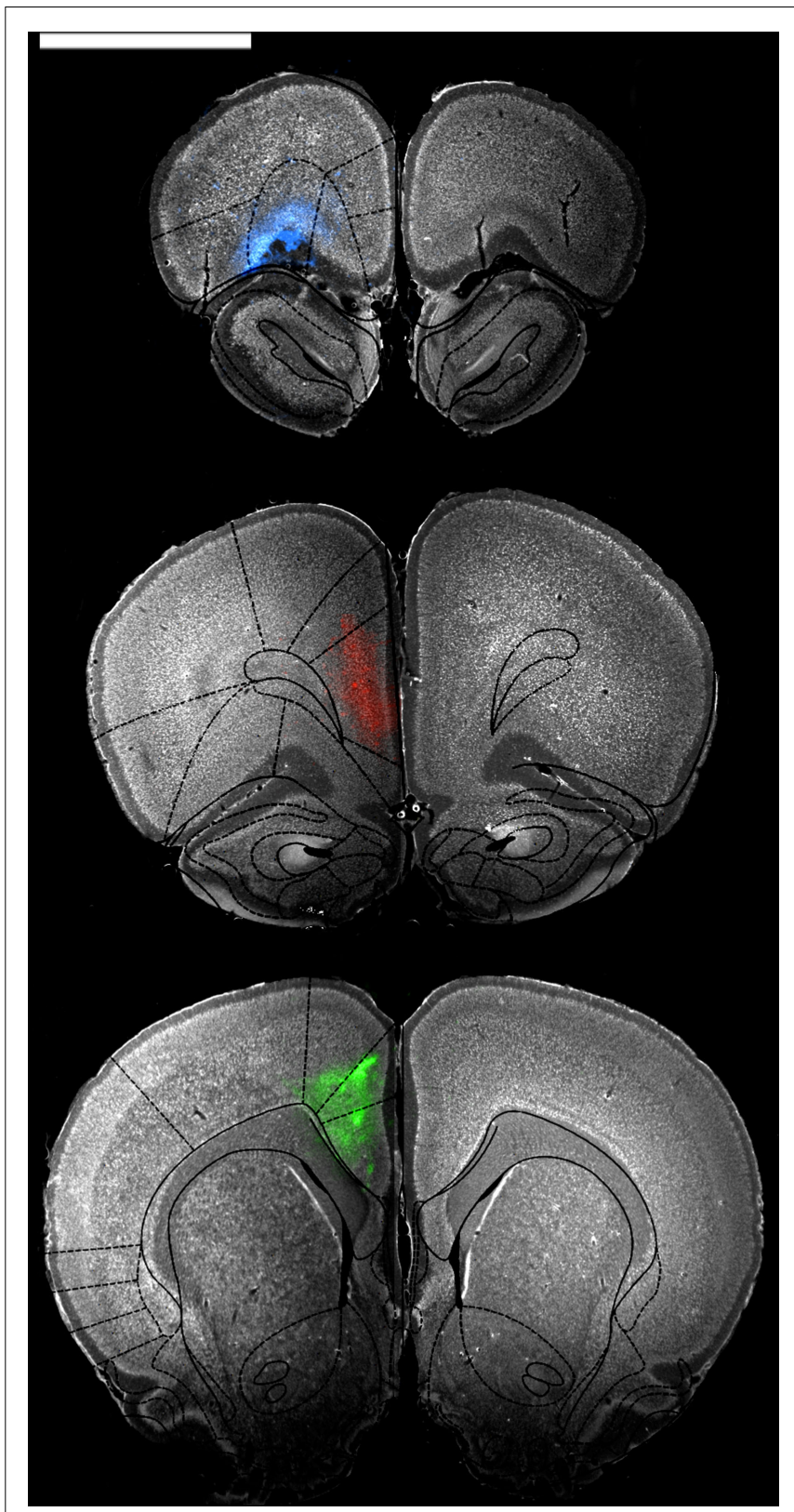

FIGURE 1 | Representative low power photomicrographs showing injections into OFC (blue, top panel), MPFC (red, middle panel), and ACC (green, bottom panel). Sections were stained with a fluorescent Nissl stain and then photographed through appropriate fluorescence filters to show cell bodies and the extent of tracer diffusion. The images were then overlaid with atlas images from Paxinos and Watson (1997) to confirm restriction of tracer to regions of interest. Scale bar $=4 \mathrm{~cm}$.

Data suggest that these nuclei are vastly different with respect to the level of collateralization of axons projecting to OFC, $\mathrm{mPFC}$, and ACC. Damage to the tissue encompassing NBM in two brains made it impossible to identify NBM, therefore data for this nucleus are representative of only seven animals. About $23.3 \%$ of retrogradely labeled putative cholinergic cells were found to collateralize to all three prefrontal subregions, $37.4 \%$ projected to any two 

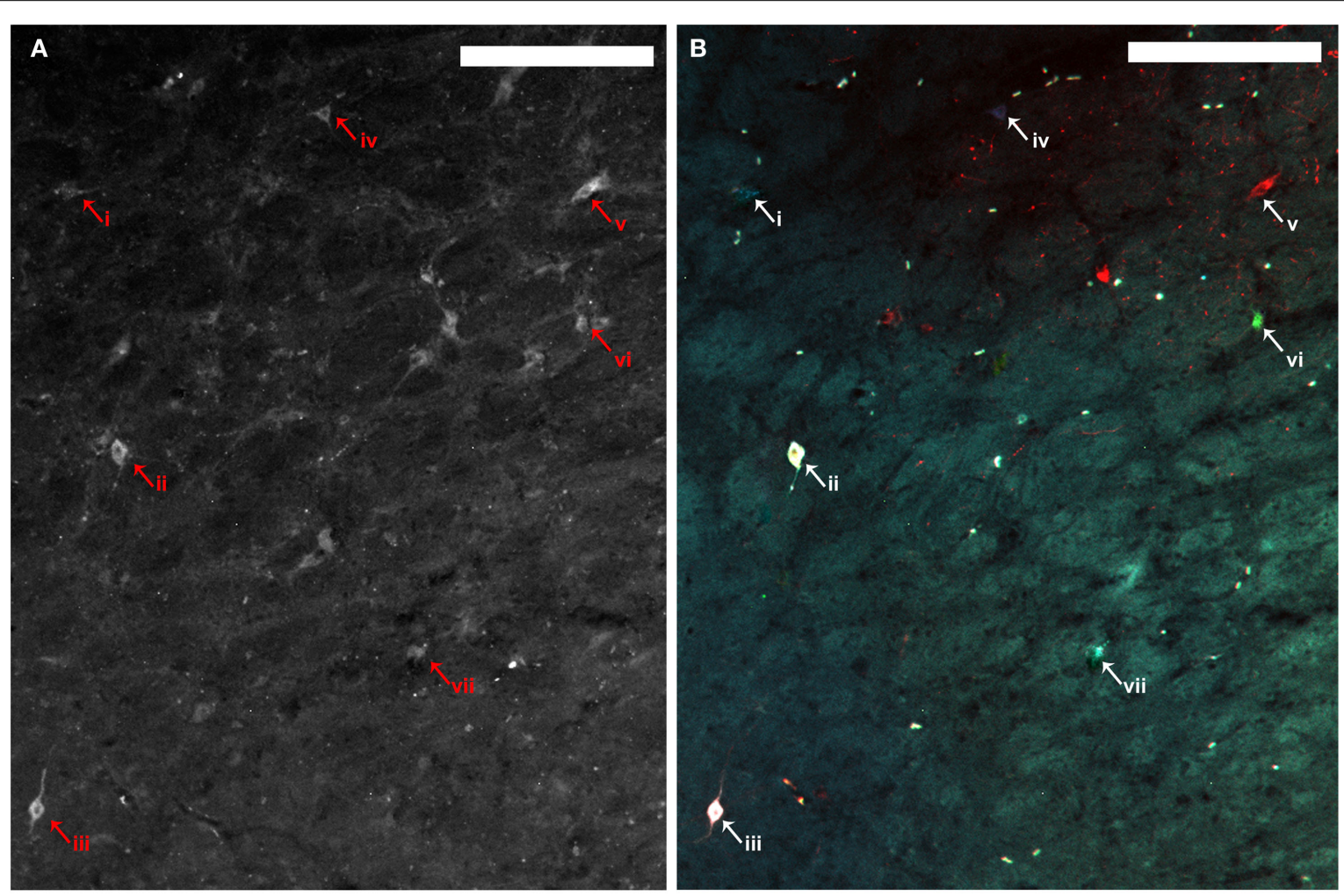

C
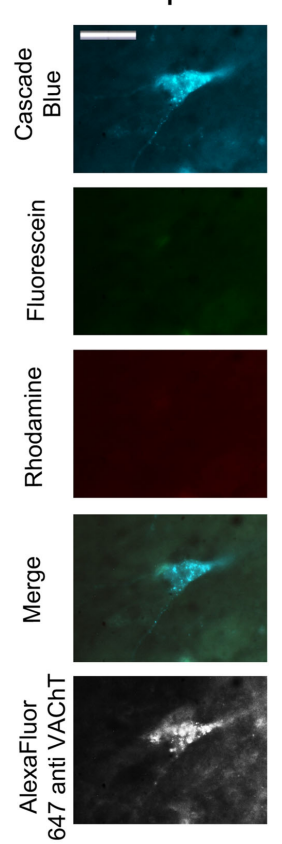

ii
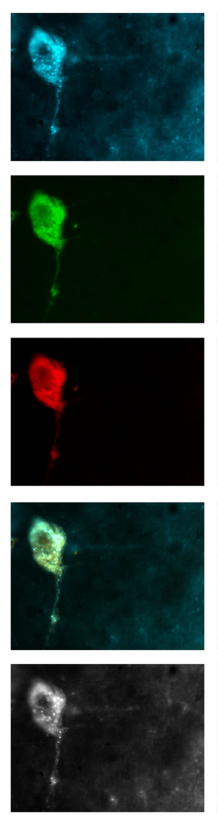

iii
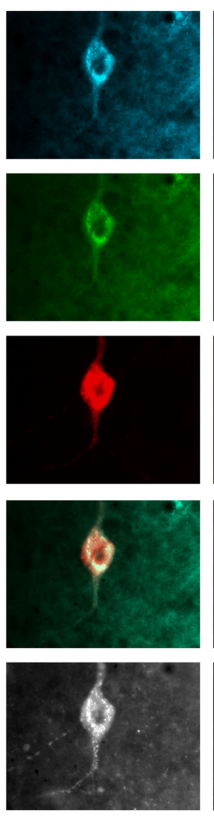

FIGURE 2 | (A,B) Show the same representative mid-level NBM section through the fluorescence channel to detect VAChT immunofluorescence (A) and merged fluorescence channels to detect retrograde tracer (B). Scale bars $=200 \mu \mathrm{m}$. (C) shows high power images of cells represented by arrowheads and Roman numerals in (A,B). The top three rows illustrate each image through the fluorescence channels necessary to detect the three retrograde tracers. Images in row four show the three composite images merged with one another and demonstrate which tracers individual cells contained, and therefore whether or not they projected to multiple PFC terminal fields. The same cells are shown through the fluorescence channel to detect VAChT immunofluorescence in the bottom row. Scale bars in (C) $=20 \mu \mathrm{m}$. VAChT: vesicular acetylcholine transporter. 

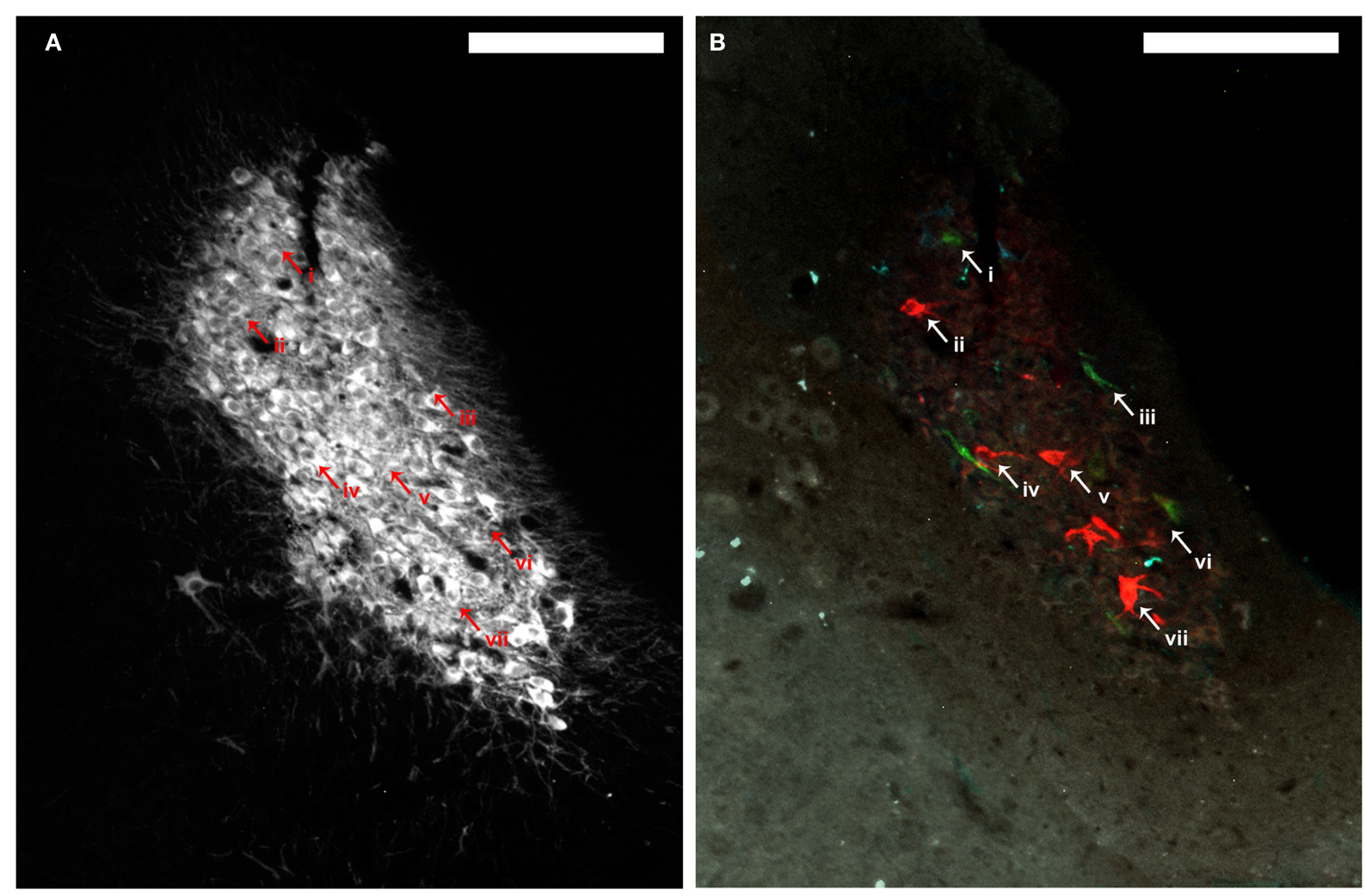

C
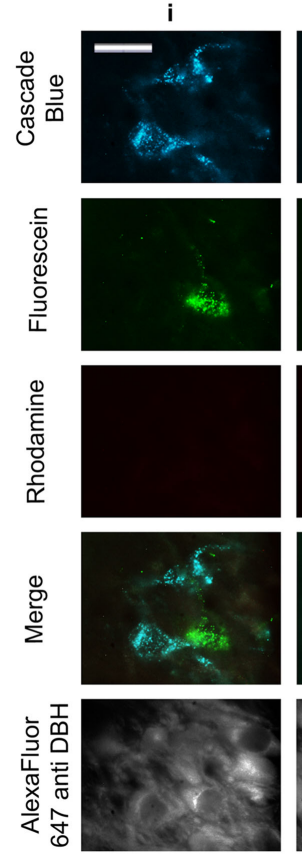

ii
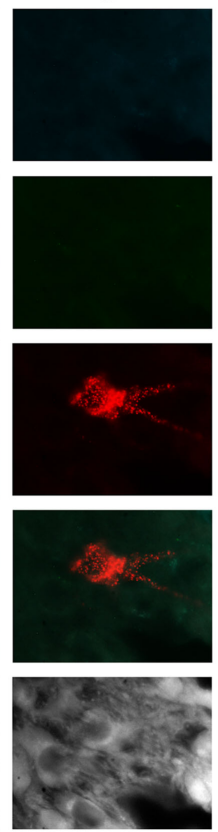

iii
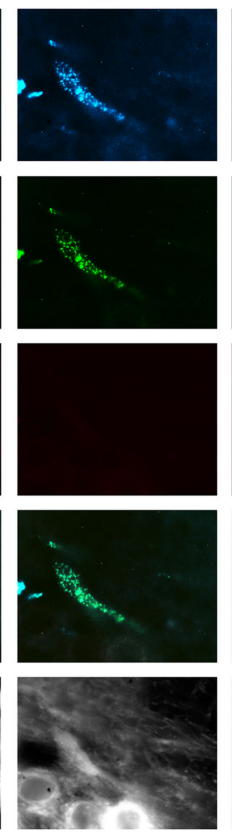

FIGURE 3 | (A,B) Show the same representative LC section through the fluorescence channel to detect $\mathrm{DBH}(\mathbf{A})$ and merged fluorescence channels to detect retrograde tracer (B). Scale bars $=200 \mu \mathrm{m}$. (C) shows high power images of cells represented by arrowheads and Roman numerals in $\mathbf{( A , B )}$. The top three rows illustrate each image through the fluorescence channels necessary to detect the three retrograde tracers. iv
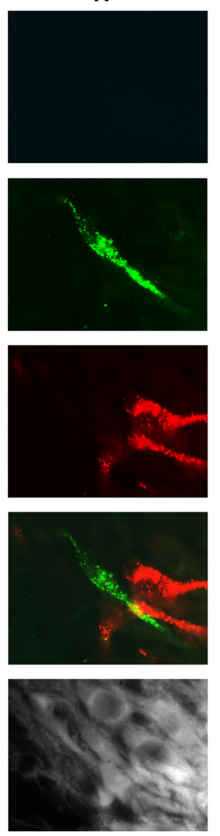

v
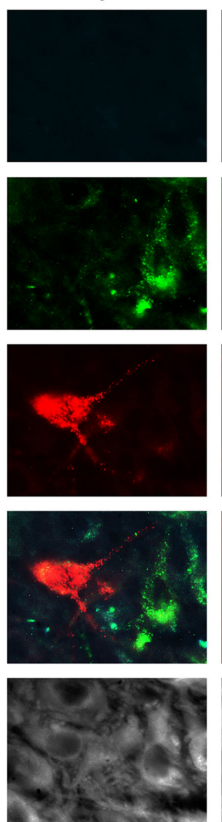

vi
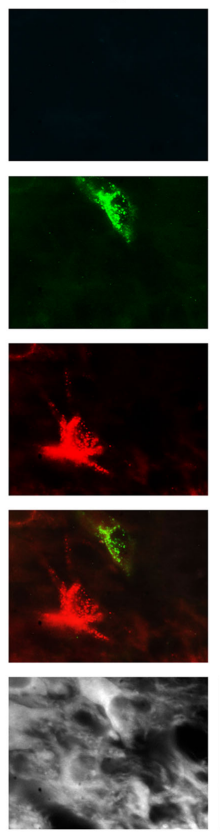

vii
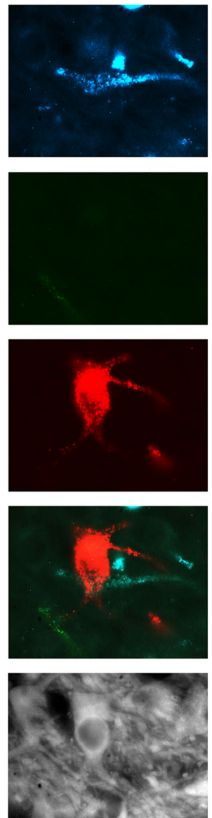

Images in row four show the three composite images merged with one another and demonstrate which tracers individual cells contained, and therefore whether or not they projected to multiple PFC terminal fields. The same cells are shown through the fluorescence channel to detect $\mathrm{DBH}$ immunofluorescence in the bottom row. Scale bars in $\mathbf{( C )}=20 \mu \mathrm{m}$. $\mathrm{DBH}$ : dopamine $\beta$ hydroxylase. 
subregions, and $39.3 \%$ projected to any single target. In LC $(n=9)$, however, only $4.3 \%$ of retrogradely labeled neurons possessed collaterals targeting any two prefrontal subregions simultaneously, and no cells were identified that projected to all three regions. All remaining cells were single labeled and therefore exhibited a restricted axonal terminal field with respect to individual PFC subregions (Figure 4). These data suggest that assemblies of NBM neurons possess highly divergent axons capable of coordinating ACh release in multiple PFC subregions simultaneously (as well as in any other terminal fields to which these axons collateralize). LC efferents, on the other hand, appear to be organized so as to elicit NE release in only one of these PFC subregions at a time. Interestingly, some topographic order was also identified for these projection neurons in NBM and LC. Although no differences
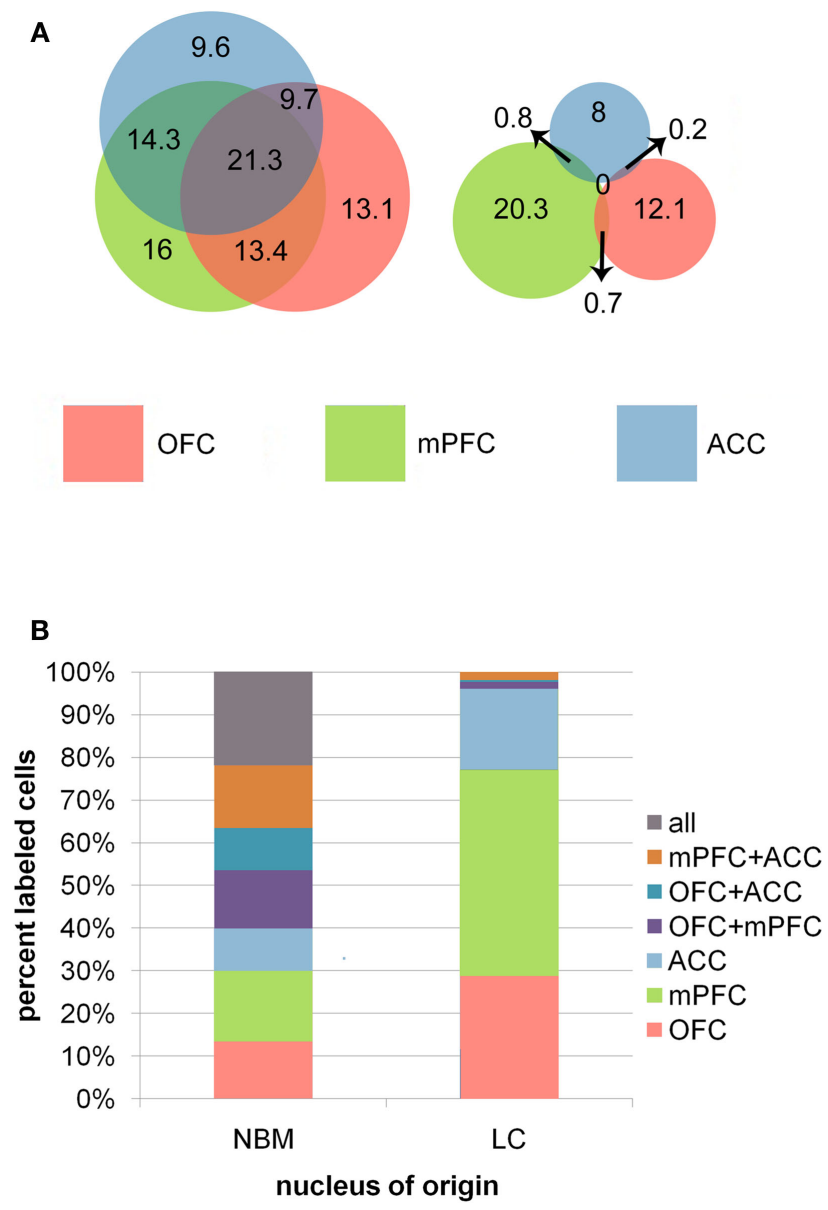

FIGURE 4 | (A) Venn diagrams representing the relative sizes and degree of overlap between populations of projection neurons in NBM and LC. Overlapping regions correspond to populations of cells that projected to multiple PFC terminal field regions. Note the high degree of overlap between in NBM, representing a high proportion of double and triple labeled cells in this nucleus, whereas LC had very minimal overlap between the three populations of projection cells. Numbers indicate the mean number of cells per animal identified within each population. (B) The bar graphs illustrate the percentages of total retrogradely labeled neurons in NBM and LC that project to each possible combination of targets within the PFC. existed between populations of cells projecting to the different possible combinations of PFC subregions in either nucleus, PFC projection cells in NBM clustered in the rostral half of the nucleus, and those in LC were restricted to its compact core and never localized within the rostral or caudal poles. The projection cells were almost always labeled ipsilateral to the injection sites. Therefore, the neurons projecting to OFC, $\mathrm{mPFC}$, and ACC do so primarily ipsilaterally, and although they do cluster within specific zones of each nucleus, they are interdigitated with one another rather than spatially segregated.

These broad versus specific terminal field organizations may dictate discrete roles for cholinergic and noradrenergic systems in cognitive function. For example, coordinated ACh release within several subregions of PFC may facilitate the transmission of behaviorally relevant signals across multiple PFC networks simultaneously in order to evaluate the salience of this information and promote optimal decision making. It has been shown that ACh release in neocortex generally promotes vigilance and environmental awareness by desynchronizing slow cortical EEG patterns, and also increases the signal to noise ratio of sensory inputs (Wenk, 1997). Furthermore, ACh acts on both GABAergic and glutamatergic systems in cortex to suppress weaker inputs to a greater extent than strong inputs (Metherate et al., 1992; Metherate and Ashe, 1995a,b), which may prepare PFC networks to respond to salient stimuli that elicit large responses while filtering out behaviorally irrelevant stimuli that do not. Consider again the example of the go/no-go task. The simultaneous release of ACh within multiple PFC subregions may alter response thresholds of individual neurons and prime the networks to respond to the relevant stimulus (a red square), even in the absence of its physical presentation. In this way, an animal may prepare to identify the correct stimulus when it appears in an unexpected location, while simultaneously ignoring irrelevant stimuli.

Locus coeruleus, on the other hand, may serve an opposing role. The adaptive gain theory of NE function (Aston-Jones and Cohen, 2005a,b) suggests that during optimal behavioral performance, LC neurons are capable of acting as independent units and modulating restricted terminal field regions to promote exploitation of the current behavioral strategy. Then, as behavioral performance suddenly decreases (as it does during unexpected uncertainty), LC neurons begin to fire tonically en masse through dendritic electrotonic coupling, orchestrating a more homogeneous effect on network properties throughout all terminal field regions of LC. In this state, animals are less focused on specific stimuli but rather operate in a scanning mode, searching the environment in order to identify new relevant stimuli. As new behaviorally relevant targets are identified, LC neurons once again begin to fire independently with phasic discharges, thus promoting a new behavioral strategy. Such plasticity of the firing properties and responsivity of LC neurons are well documented (Aston-Jones et al., 1994, 2000; Dalley et al., 2001, 2004). The restricted pattern of LC projections to PFC subregions as observed in our work fits well with this theory: Independent modulation of prefrontal subregions to promote dissociable behavioral operations of these areas would depend on the existence of subsets of LC neurons that project with exclusivity to restricted PFC targets. Then, during a decrease in the utility of an ongoing behavioral strategy, LC neurons would begin to fire in 
unison, leading to greater synchrony of NE release across regions, thereby disrupting focused attention in favor of scanning attention and broadly tuned signal processing.

These anatomical findings of the efferent NBM and LC projections to PFC reflect their unique roles in cognitive function. Coordinated ACh release in subregions of PFC during ideal behavioral performance may prime the networks to focus on or become sensitized to specific stimuli, even if the stimuli are physically absent. In this way, these divergent neurons help to maintain network properties across broad areas of cortex so that an animal can respond when a relevant stimulus does occur in an unexpected location or time. The restricted nature of LC axons projecting to OFC, $\mathrm{mPFC}$, and ACC, on the other hand, may facilitate the exploitation of a behavioral strategy that is uniquely supported by one of these regions by specifically altering only its network activity. It is known that these regions of PFC project back down to LC (Aston-Jones and Cohen, 2005a,b), perhaps to signal to the nucleus that an ongoing behavioral strategy is in fact paying off and facilitate the maintenance of its current firing mode. When particular cells in LC receive this information, they may then cause subsequent NE release in that same area to continue facilitating its behavioral function. As unexpected uncertainty arises, or a sudden change in stimulus value occurs, LC begins to fire tonically en masse (perhaps as a result of a loss of or change in input from PFC), eliciting NE release in all terminal fields, pushing the animal's attentional state to scan its environment for previously ignored stimuli that may now be relevant. When the novel reinforcement contingency is identified, global ACh release may again begin to occur, as well as restricted NE release to sensitize the necessary networks to the novel target stimulus and exploit the new behavioral strategy.

The present findings are strictly anatomical in nature. They do, however, accord well with computational, behavioral, and

\section{REFERENCES}

Arendt, T., Bigl, V., Arendt, A., and Tennstedt, A. (1983). Loss of neurons in the nucleus basalis of Meynert in Alzheimer's disease, paralysis agitans and Korsakoff's disease. Acta Neuropathol. 61, 101-108.

Arnsten, A. F. (2000). Through the looking glass: differential noradenergic modulation of prefrontal cortical function. Neural Plast. 7, 133-146.

Arnsten, A. F., and Pliszka, S. R. (2011). Catecholamine influences on prefrontal cortical function: relevance to treatment of attention deficit/hyperactivity disorder and related disorders. Pharmacol. Biochem. Behav. 99, 211-216.

Aston-Jones, G., and Cohen, J. D. (2005a). Adaptive gain and the role of the locus coeruleusnorepinephrine system in optimal performance. J. Comp. Neurol. 493, 99-110.

Aston-Jones, G., and Cohen, J. D. (2005b). An integrative theory of locus coeruleus-norepinephrine function: adaptive gain and optimal performance. Annu. Rev. Neurosci. 28, 403-450.

Aston-Jones, G., Rajkowski, J., and Cohen, J. (2000). Locus coeruleus and regulation of behavioral flexibility and attention. Prog. Brain Res. 126, 165-182.

Aston-Jones, G., Rajkowski, J., Kubiak, P., and Alexinsky, T. (1994). Locus coeruleus neurons in monkey are selectively activated by attended cues in a vigilance task. J. Neurosci. 14, 4467-4480.

Berridge, C. W., and Waterhouse, B. D. (2003). The locus coeruleusnoradrenergic system: modulation of behavioral state and statedependent cognitive processes. Brain Res. Brain Res. Rev. 42, 33-84.

Birrell, J. M., and Brown, V. J. (2000). Medial frontal cortex mediates perceptual attentional set shifting in the rat. J. Neurosci. 20, 4320-4324.

pharmacological studies of the cholinergic and noradrenergic systems in modulation of cognition and executive function. Future studies may further explore these anatomical distributions of PFC projection neurons in the contexts of normal developmental trajectories as well as animal models of psychiatric and neurodegenerative disease in order to correlate change in behavioral performance with a change in nuclear organization and distribution. It is equally important to characterize the $\mathrm{NE}$ and $\mathrm{ACh}$ receptor distributions within these PFC terminal field subregions. As each of these transmitters interacts with a number of membrane bound receptors, their ultimate effect on network properties depends not only on neurotransmitter release, but also on receptor binding and receptor expression. Different environmental and behavioral circumstances may promote engagement of different receptor subtypes; such information is necessary to gain a more complete understanding of the role of these modulatory systems in PFC function. Likewise, the precise nature of the downstream projections from PFC subregions to NBM and LC remains to be elucidated. For example, the demonstration of a PFC subregion specific afferent topography within these nuclei would further support a highly ordered and functional role for these modulatory systems in cognition and behavior. Characterization and manipulation of the NBM and LC projection neurons at biochemical, genetic, pharmacological and physiological levels will provide a comprehensive and multi-faceted analysis of these cells and their role in regulating prefrontal local circuit operations and behavioral outcomes. Such studies may inform the development of novel and more effective therapeutics for psychiatric and neurodegenerative diseases associated with dysfunction of these ascending modulatory pathways.

\section{ACKNOWLEDGMENTS}

The experimental portions of this work are supported by NIDA DA017960 and NIMH MH087921.

Bissonette, G. B., Martins, G. J., Franz, T. M., Harper, E. S., Schoenbaum, G., and Powell, E. M. (2008). Double dissociation of the effects of medial and orbital prefrontal cortical lesions on attentional and affective shifts in mice. J. Neurosci. 28, 11124-11130.

Bondareff, W., Mountjoy, C. Q., and Roth, M. (1982). Loss of neurons of origin of the adrenergic projection to cerebral cortex (nucleus locus ceruleus) in senile dementia. Neurology 32, 164-168.

Bouret, S., and Sara, S. J. (2004). Reward expectation, orientation of attention and locus coeruleus-medial frontal cortex interplay during learning. Eur. J. Neurosci. 20, 791-802.

Bouret, S., and Sara, S. J. (2005). Network reset: a simplified overarching theory of locus coeruleus noradrenaline function. Trends Neurosci. 28, 574-582.

Bucci, D. J., Holland, P. C., and Gallagher, M. (1998). Removal of cholinergic input to rat posterior parietal cortex disrupts incremental processing of conditioned stimuli. $J$. Neurosci. 18, 8038-8046.

Bussey, T. J., Everitt, B. J., and Robbins, T. W. (1997a). Dissociable effects of cingulate and medial frontal cortex lesions on stimulus-reward learning using a novel Pavlovian autoshaping procedure for the rat: implications for the neurobiology of emotion. Behav. Neurosci. 111, 908-919.

Bussey, T. J., Muir, J. L., Everitt, B. J., and Robbins, T. W. (1997b). Triple dissociation of anterior cingulate, posterior cingulate, and medial frontal cortices on visual discrimination tasks using a touchscreen testing procedure for the rat. Behav. Neurosci. 111, 920-936.

Cain, R. E., Wasserman, M. C., Waterhouse, B. D., and McGaughy, J. A. (2011). Atomoxetine facilitates Attentional Set Shifting in adolescent rats. Dev. Cogn. Neurosci. 1, 552-559. 
Cardinal, R. N., Parkinson, J. A., Lachenal, G., Halkerston, K. M., Rudarakanchana, N., Hall, J., Morrison, C. H., Howes, S. R., Robbins, T. W., and Everitt, B. J. (2002). Effects of selective excitotoxic lesions of the nucleus accumbens core, anterior cingulate cortex, and central nucleus of the amygdala on autoshaping performance in rats. Behav. Neurosci. $116,553-567$.

Chiba, A. A., Bucci, D. J., Holland, P. C., and Gallagher, M. (1995). Basal forebrain cholinergic lesions disrupt increments but not decrements in conditioned stimulus processing. $J$. Neurosci. 15, 7315-7322.

Dalley, J. W., Cardinal, R. N., and Robbins, T. W. (2004). Prefrontal executive and cognitive functions in rodents: neural and neurochemical substrates. Neurosci. Biobehav. Rev 28, 771-784.

Dalley, J. W., McGaughy, J., O'Connell, M. T., Cardinal, R. N., Levita, L., and Robbins, T. W. (2001). Distinct changes in cortical acetylcholine and noradrenaline efflux during contingent and noncontingent performance of a visual attentional task. J. Neurosci. 21, 4908-4914.

Devilbiss, D. M., Page, M. E., and Waterhouse, B. D. (2006). Locus ceruleus regulates sensory encoding by neurons and networks in waking animals. J. Neurosci. 26, 9860-9872.

Devilbiss, D. M., and Waterhouse, B. D. (2000). Norepinephrine exhibits two distinct profiles of action on sensory cortical neuron responses to excitatory synaptic stimuli. Synapse 37, 273-282.

Devilbiss, D. M., and Waterhouse, B. D. (2004). The effects of tonic locus ceruleus output on sensoryevoked responses of ventral posterior medial thalamic and barrel field cortical neurons in the awake rat. $J$. Neurosci. 24, 10773-10785.

Dias, R., Robbins, T. W., and Roberts, A. C. (1996). Dissociation in prefrontal cortex of affective and attentional shifts. Nature 380, 69-72.

Dias, R., Robbins, T. W., and Roberts, A. C. (1997). Dissociable forms of inhibitory control within prefrontal cortex with an analog of the Wisconsin Card Sort Test: restriction to novel situations and independence from "on-line" processing. $J$. Neurosci. 17, 9285-9297.

Fallon, J. H., and Loughlin, S. E. (1982). Monoamine innervation of the forebrain: collateralization. Brain Res. Bull. 9, 295-307.

Gamo, N. J., and Arnsten, A. F. (2011). Molecular modulation of prefrontal cortex: rational development of treatments for psychiatric disorders. Behav. Neurosci. 125, 282-296.

Gesi, M., Soldani, P., Giorgi, F. S., Santinami, A., Bonaccorsi, I., and Fornai, F. (2000). The role of the locus coeruleus in the development of Parkinson's disease. Neurosci. Biobehav. Rev. 24, 655-668.

Hurley, L. M., Devilbiss, D. M., and Waterhouse, B. D. (2004). A matter of focus: monoaminergic modulation of stimulus coding in mammalian sensory networks. Curr. Opin. Neurobiol. 14, 488-495.

Lehmann, J., Nagy, J. I., Atmadia, S., and Fibiger, H. C. (1980). The nucleus basalis magnocellularis: the origin of a cholinergic projection to the neocortex of the rat. Neuroscience 5, 1161-1174.

Loughlin, S. E., Foote, S. L., and Fallon, J. H. (1982). Locus coeruleus projections to cortex: topography, morphology and collateralization. Brain Res. Bull. 9, 287-294.

Maddux, J. M., Kerfoot, E. C., Chatterjee, S., and Holland, P. C. (2007). Dissociation of attention in learning and action: effects of lesions of the amygdala central nucleus, medial prefrontal cortex, and posterior parietal cortex. Behav. Neurosci. 121, 63-79.

McGaughy, J., Dalley, J. W., Morrison, C. H., Everitt, B. J., and Robbins, T. W. (2002). Selective behavioral and neurochemical effects of cholinergic lesions produced by intrabasalis infusions of 192 IgG-saporin on attentional performance in a fivechoice serial reaction time task. $J$. Neurosci. 22, 1905-1913.

McGaughy, J., Everitt, B. J., Robbins, T. W., and Sarter, M. (2000). The role of cortical cholinergic afferent projections in cognition: impact of new selective immunotoxins. Behav. Brain Res. 115, 251-263.

McGaughy, J., Ross, R. S., and Eichenbaum, H. (2008). Noradrenergic, but not cholinergic, deafferentation of prefrontal cortex impairs attentional set-shifting. Neuroscience 153, 63-71.

McGaughy, J., and Sarter, M. (1995). Effects of chlordiazepoxide and scopolamine, but not aging, on the detection and identification of conditional visual stimuli. J. Gerontol. A Biol. Sci. Med. Sci. 50, B90-B96.

McGaughy, J., and Sarter, M. (1998). Sustained attention performance in rats with intracortical infusions of 192 IgG-saporin-induced cortical cholinergic deafferentation: effects of physostigmine and FG 7142. Behav. Neurosci. 112, 1519-1525.
McGaughy, J., and Sarter, M. (1999). Effects of ovariectomy, 192 IgGsaporin-induced cortical cholinergic deafferentation, and administration of estradiol on sustained attention performance in rats. Behav. Neurosci. 113, 1216-1232.

McMillan, P. J., White, S. S., Franklin, A., Greenup, J. L., Leverenz, J. B., Raskind, M. A., and Szot, P. (2011). Differential response of the central noradrenergic nervous system to the loss of locus coeruleus neurons in Parkinson's disease and Alzheimer's disease. Brain Res. 1373, 240-252.

Metherate, R., and Ashe, J. H. (1995a). GABAergic suppression prevents the appearance and subsequent fatigue of an NMDA receptor-mediated potential in neocortex. Brain Res. 699, 221-230.

Metherate, R., and Ashe, J. H. (1995b). Synaptic interactions involving acetylcholine, glutamate, and GABA in rat auditory cortex. Exp. Brain Res. 107, 59-72.

Metherate, R., Cox, C. L., and Ashe, J. H. (1992). Cellular bases of neocortical activation: modulation of neural oscillations by the nucleus basalis and endogenous acetylcholine. J. Neurosci. 12, 4701-4711.

Milstein, J. A., Lehmann, O., Theobald, D. E., Dalley, J. W., and Robbins, T. W. (2007). Selective depletion of cortical noradrenaline by antidopamine beta-hydroxylase-saporin impairs attentional function and enhances the effects of guanfacine in the rat. Psychopharmacology (Berl.) 190, 51-63.

Moxon, K. A., Devilbiss, D. M., Chapin, J. K., and Waterhouse, B. D. (2007) Influence of norepinephrine on somatosensory neuronal responses in the rat thalamus: a combined modeling and in vivo multi-channel, multi-neuron recording study. Brain Res. 1147, 105-123.

Muir, J. L., Everitt, B. J., and Robbins, T. W. (1996). The cerebral cortex of the rat and visual attentional function: dissociable effects of mediofrontal, cingulate, anterior dorsolateral, and parietal cortex lesions on a fivechoice serial reaction time task. Cereb. Cortex 6, 470-481.

Newman, L. A., Darling, J., and McGaughy, J. (2008). Atomoxetine reverses attentional deficits produced by noradrenergic deafferentation of medial prefrontal cortex. Psychopharmacology (Berl.) 200 , 39-50.

Newman, L. A., and McGaughy, J. (2008). Cholinergic deafferentation of prefrontal cortex increases sensitivity to cross-modal distractors during a sustained attention task. $J$. Neurosci. 28, 2642-2650.

Nieto-Escamez, F. A., Sanchez-Santed, F., and De Bruin, J. P. (2002). Cholinergic receptor blockade in prefrontal cortex and lesions of the nucleus basalis: implications for allocentric and egocentric spatial memory in rats. Behav. Brain Res. 134, 93-112.

Owen, A. M., Roberts, A. C., Hodges, J. R., Summers, B. A., Polkey, C. E., and Robbins, T. W. (1993). Contrasting mechanisms of impaired attentional set-shifting in patients with frontal lobe damage or Parkinson's disease. Brain 116(Pt 5), 1159-1175.

Owen, A. M., Roberts, A. C., Polkey, C. E., Sahakian, B. J., and Robbins, T. W. (1991). Extra-dimensional versus intra-dimensional set shifting performance following frontal lobe excisions, temporal lobe excisions or amygdalo-hippocampectomy in man. Neuropsychologia 29, 993-1006.

Pang, K., Williams, M. J., Egeth, H., and Olton, D. S. (1993). Nucleus basalis magnocellularis and attention: effects of muscimol infusions. Behav. Neurosci. 107, 1031-1038.

Pantelis, C., Barber, F. Z., Barnes, T. R. Nelson, H. E., Owen, A. M., and Robbins, T. W. (1999). Comparison of set-shifting ability in patients with chronic schizophrenia and frontal lobe damage. Schizophr. Res. 37, 251-270.

Parkinson, J. A., Willoughby, P. J., Robbins, T. W., and Everitt, B. J. (2000). Disconnection of the anterior cingulate cortex and nucleus accumbens core impairs Pavlovian approach behavior: further evidence for limbic cortical-ventral striatopallidal systems. Behav. Neurosci. 114, 42-63.

Paxinos, G., and Watson, C. (1997). The Rat Brain, in Stereotaxic Coordinates. San Diego: Academic Press.

Rahman, S., Robbins, T. W., Hodges, J. R., Mehta, M. A., Nestor, P. J., Clark, L., and Sahakian, B. J. (2006). Methylphenidate ('Ritalin') can ameliorate abnormal risk-taking behavior in the frontal variant of frontotemporal dementia. Neuropsychopharmacology 31, 651-658.

Ramos, B. P., and Arnsten, A. F. (2007). Adrenergic pharmacology and cognition: focus on the prefrontal cortex. Pharmacol. Ther. 113, 523-536.

Robbins, T. W. (2000). Chemical neuromodulation of frontal-executive functions in humans and other animals. Exp. Brain Res. 133 130-138. 
Sara, S. J. (2009). The locus coeruleus and noradrenergic modulation of cognition. Nat. Rev. Neurosci. 10, 211-223.

Sarter, M., and Bruno, J. P. (2000). Cortical cholinergic inputs mediating arousal, attentional processing and dreaming: differential afferent regulation of the basal forebrain by telencephalic and brainstem afferents. Neuroscience 95, 933-952.

Simpson, K. L., Altman, D. W., Wang, L., Kirifides, M. L., Lin, R. C., and Waterhouse, B. D. (1997). Lateralization and functional organization of the locus coeruleus projection to the trigeminal somatosensory pathway in rat. J. Comp. Neurol. 385, 135-147.

Simpson, K. L., Waterhouse, B. D., and Lin, R. C. (1999). Origin, distribution, and morphology of galaninergic fibers in the rodent trigeminal system. J. Comp. Neurol. 411, 524-534.
Simpson, K. L., Waterhouse, B. D., and Lin, R. C. (2006). Characterization of neurochemically specific projections from the locus coeruleus with respect to somatosensoryrelated barrels. Anat. Rec. A Discov. Mol. Cell. Evol. Biol. 288, 166-173.

Von Coelln, R., Thomas, B., Savitt, J. M., Lim, K. L., Sasaki, M., Hess, E. J., Dawson, V. L., and Dawson, T. M. (2004). Loss of locus coeruleus neurons and reduced startle in parkin null mice. Proc. Natl. Acad. Sci. U.S.A. 101, 10744-10749.

Wallace, T. L., Ballard, T. M., Pouzet, B., Riedel, W. J., and Wettstein, J. G. (2011). Drug targets for cognitive enhancement in neuropsychiatric disorders. Pharmacol. Biochem. Behav. 99, 130-145.

Wallace, T. L., and Porter, R. H. (2011). Targeting the nicotinic alpha7 acetylcholine receptor to enhance cognition in disease. Biochem. Pharmacol. 82, 891-903.

Waterhouse, B. D., Border, B., Wahl, L. and Mihailoff, G. A. (1993). Topographic organization of rat locus coeruleus and dorsal raphe nuclei: distribution of cells projecting to visual system structures. J. Comp. Neurol. 336, 345-361.

Waterhouse, B. D., Lin, C. S., Burne, R. A., and Woodward, D. J. (1983). The distribution of neocortical projection neurons in the locus coeruleus. J. Comp. Neurol. 217, 418-431.

Wenk, G. L. (1997). The nucleus basalis magnocellularis cholinergic system: one hundred years of progress. $\mathrm{Neu}$ robiol. Learn. Mem. 67, 85-95.

Yu, A. J., and Dayan, P. (2002). Acetylcholine in cortical inference. Neural. Netw. 15, 719-730.

Yu, A. J., and Dayan, P. (2005). Uncertainty, neuromodulation, and attention. Neuron 46, 681-692.
Conflict of Interest Statement: The authors declare that the research was conducted in the absence of any commercial or financial relationships that could be construed as a potential conflict of interest.

Received: 10 February 2012; accepted: 19 April 2012; published online: 21 May 2012.

Citation: Chandler D and Waterhouse $B D$ (2012) Evidence for broad versus segregated projections from cholinergic and noradrenergic nuclei to functionally and anatomically discrete subregions of prefrontal cortex. Front. Behav. Neurosci. 6:20. doi: 10.3389/fnbeh.2012.00020 Copyright (C) 2012 Chandler and Waterhouse. This is an open-access article distributed under the terms of the Creative Commons Attribution Non Commercial License, which permits noncommercial use, distribution, and reproduction in other forums, provided the original authors and source are credited. 